PROCEEDINGS OF THE AMERICAN MATHEMATICAL SOCIETY

Volume 124, Number 11, November 1996

\title{
ON THE PICARD GROUP OF A COMPACT FLAT PROJECTIVE VARIETY
}

\author{
N. J. MICHELACAKIS
}

(Communicated by Peter Li)

\begin{abstract}
In this note, we describe the Picard group of the class of compact, smooth, flat, projective varieties. In view of Charlap's work and Johnson's characterization, we construct line bundles over such manifolds as the holonomy-invariant elements of the Neron-Severi group of a projective flat torus covering the manifold. We prove a generalized version of the AppellHumbert theorem which shows that the nontrivial elements of the Picard group are precisely those coming from the above construction. Our calculations finally give an estimate for the set of positive line bundles for such varieties.
\end{abstract}

\section{INTRODUCTION}

Let $M$ denote a compact, connected, complex, flat Riemannian manifold. In 1912 Bieberbach proved that every compact flat Riemannian manifold $M$ is finitely covered by a flat torus. For a proof, see e.g. [14]. Furthermore, these manifolds are classified [3], up to connection preserving diffeomorphisms, by associating to $M$ a short exact sequence

$$
0 \longrightarrow \Lambda \longrightarrow G \longrightarrow \Phi \longrightarrow 1
$$

in which $\Phi$, the holonomy group of $M$, is finite and $\Lambda \simeq \mathbf{Z}^{2 n}$ is the translation subgroup of $G \simeq \pi_{1}(M)$, a torsion free, discrete, cocompact subgroup of $\mathcal{M}_{2 n}$, the group of Euclidean motions of $\mathbf{R}_{n}$. Conversely, given any such torsion free extension, $G$ imbeds as a discrete cocompact subgroup of $\mathcal{M}_{2 n}$, and $M$ is isometric to $G \backslash \mathbf{E}_{n} / O_{2 n}$, where $O_{2 n}$ is the isotropy group of the origin.

We let $V \simeq V^{t}$ denote the complex vector space of dimension $n$ and complex structure $t$ whose underlying real vector space is $\Lambda \otimes_{\mathbf{z}} \mathbf{R}$. Further, let $\rho$ be a faithful integral representation $\rho: \Phi \longrightarrow \mathrm{GL}_{\mathbf{Z}}(\Lambda)$ and denote by $\Lambda^{\rho}$ and $V^{\rho_{R}}$ the $\mathbf{Z}[\Phi]$ and $\mathbf{R}[\Phi]$-module where $\Phi$ acts by means of $\rho$ and $\rho_{\mathbf{R}}: \Phi \rightarrow \operatorname{GL}_{\mathbf{Z}}(\Lambda) \hookrightarrow \mathrm{GL}_{\mathbf{R}}(V)$ respectively.

When there is no fear of confusion we may write $\Lambda$ instead of $\Lambda^{\rho}$, and $V$ instead of $V^{\rho_{R}}$. We shall say $V$ admits a complex structure if there exists a map $t \in$ $\operatorname{End}_{\mathbf{R}[\Phi]}\left(V^{\rho_{R}}\right)$ such that $t^{2}=1$. In view of the well known correspondence between

Received by the editors May 22, 1995.

1991 Mathematics Subject Classification. Primary 14C22; Secondary 14A10, 14E20.

Key words and phrases. Appell-Humbert theorem, Bieberbach group, cohomology of groups, complex structure, group action, global section of line bundle, group representation, flat Riemannian manifold, holonomy group, Lyndon-Hotschild-Serre spectral sequence, Lefschetz's theorem, Lie group, (ample) line bundle, Neron-Severi group, Picard group.

(C)1996 American Mathematical Society 
$\Phi$-representations over a ring $K$ and $K[\Phi]$-modules, we can, in the language of representation theory, equivalently define $t$ to be a complex structure for $(\Lambda, \rho)$ when $\operatorname{Im}(\rho) \subset \mathrm{GL}_{\mathbf{C}}\left(V^{t}\right) . \mathrm{GL}_{\mathbf{C}}\left(V^{t}\right)$ denotes the following set:

$$
\mathrm{GL}_{\mathbf{C}}\left(V^{t}\right):=\left\{m \in \mathrm{GL}_{\mathbf{R}}(V) \text { such that } m t=t m\right\} \text {. }
$$

One says that $\Lambda$ or $(\Lambda, \rho)$ admits a projective structure if, in addition to its complex structure, the complex torus $V^{t} / \Lambda$ is projective as a complex manifold. F.E.A. Johnson has given in [7] the following description for the class $\mathcal{P}$ of fundamental groups of smooth, flat, complex, projective varieties.

Theorem 1.1. $\mathcal{P}$ consists precisely of those torsion free groups $G$ which occur in an extension

$$
0 \longrightarrow \Lambda \longrightarrow G \longrightarrow \Phi \longrightarrow 1
$$

in which the operator homomorphism $\rho$ admits a $\mathcal{P}$-structure and $\Phi$ is finite.

In the second part of this paper, we study the long exact cohomology sequence of a compact, complex, flat manifold. We prove that apart from torsion, $H^{2}(M ; \mathbf{Z})$ is precisely what it was expected to be, i.e. $\left[H^{2}(\hat{M} ; \mathbf{Z})\right]^{\Phi}$, where $\hat{M} \equiv V / \Lambda$. In Proposition 3.2 of the third and final part of this paper, we construct a line bundle over $M$ whose Chern class is a given element of $[\mathrm{NS} \hat{M}]^{\Phi}$. As a corollary we get the generalized Appell-Humbert Theorem 3.4. Combining the results in sections two and three, one readily has a calculable estimate of the size of the set of all positive line bundles over $M$, this set sitting inside a free abelian group of rank $\leq \operatorname{rank}_{\mathbf{Z}}\left[H^{2}(\hat{M} ; \mathbf{Z})\right]^{\Phi}=\operatorname{dim}_{\mathbf{Q}}\left[H^{2}(\Lambda ; \mathbf{Q})\right]^{\Phi}$.

\section{The LONG COHOMOlOGY SEQUence OF A COMPACT COMPLEX FLAT MANIFOLD}

Let $\mathbf{Z}_{\mathbf{s}}, \mathcal{O}_{\mathbf{s}}, \mathcal{O}_{\mathbf{s}}^{*}$ be the sheaves of locally constant $\mathbf{Z}$-valued, holomorphic, and non-vanishing holomorphic functions on $M$. The short exact sequence of sheaves

$$
0 \longrightarrow \mathbf{Z}_{\mathbf{s}} \stackrel{j}{\longrightarrow} \mathcal{O}_{\mathbf{s}} \stackrel{e}{\longrightarrow} \mathcal{O}_{\mathbf{s}}^{*} \longrightarrow 0
$$

where $[j(f)](x)=2 \pi i f(x)$ and $[e(g)](x)=\exp g(x)$, with $x \in M$ and $f, g \in \mathbf{Z}, \mathcal{O}$ respectively, gives rise to a long exact sequence in cohomology

$$
\begin{gathered}
0 \longrightarrow H^{0}\left(M ; \mathbf{Z}_{\mathbf{s}}\right) \longrightarrow H^{0}\left(M ; \mathcal{O}_{\mathbf{s}}\right) \longrightarrow H^{0}\left(M ; \mathcal{O}_{\mathbf{s}}^{*}\right) \longrightarrow \\
H^{1}\left(M ; \mathbf{Z}_{\mathbf{s}}\right) \longrightarrow H^{1}\left(M ; \mathcal{O}_{\mathbf{s}}\right) \stackrel{e^{*}}{\longrightarrow} H^{1}\left(M ; \mathcal{O}_{\mathbf{s}}^{*}\right) \longrightarrow H^{2}(M ; \mathbf{Z}) \longrightarrow \cdots
\end{gathered}
$$

Remark 2.1. It is standard knowledge that for a simplicial complex with underlying topological space $M$, sheaf cohomology with $\mathbf{Z}_{\mathbf{s}}$ coefficients is equivalent to simplicial or even group cohomology with $\mathbf{Z}$ coefficients. Further, $M$ being a flat manifold is of the form $\mathbf{R}^{2 n} / G$, where $G$ is a Bieberbach group and therefore discrete acting freely and discontinuously on $\mathbf{R}^{2 n}$. Thus, following the appendix of [10, page 22] or alternatively $\left[6\right.$, chapter $\mathrm{V}$, page 195], we can freely replace $\mathcal{O}_{\mathbf{s}}\left[\right.$ resp. $\left.\mathcal{O}_{\mathbf{s}}^{*}\right]$ with $\mathcal{O}$ [resp. $\mathcal{O}^{*}$ ], where $\mathcal{O}$ is the $\mathbf{C}$-algebra

$$
\mathcal{O}:=\{f \mid f: V \rightarrow \mathbf{C}, f \text { holomorphic }\},
$$

and

$$
\mathcal{O}^{*}:=\left\{f \mid f: V \rightarrow \mathbf{C}^{*}, f \text { holomorphic }\right\}
$$


its group of units. So, in the case where $M$ is a flat manifold, $G$ is obviously the Bieberbach group $\pi_{1}(M)$ and $H^{*}\left(M ; \mathbf{Z}_{\mathbf{s}}\right) \simeq H^{*}\left(\pi_{1}(M) ; \mathbf{Z}\right), H^{*}\left(M ; \mathcal{O}_{\mathbf{s}}\right) \simeq$ $H^{*}\left(\pi_{1}(M) ; \mathcal{O}\right)\left[\operatorname{resp} . H^{*}\left(M ; \mathcal{O}_{\mathbf{s}}{ }^{*}\right) \simeq H^{*}\left(\pi_{1}(M) ; \mathcal{O}^{*}\right)\right]$.

Furthermore, $H^{0}(M, \mathbf{Z})=H^{0}\left(M, \mathbf{Z}_{\mathbf{s}}\right)=\Gamma\left(M, \mathbf{Z}_{\mathbf{s}}\right)=\mathbf{Z}$, and as an immediate consequence of the fact that global holomorphic functions on $M$ are constant, we get $H^{0}(M, \mathcal{O})=\mathbf{C}$ and $H^{0}\left(M, \mathcal{O}^{*}\right)=\mathbf{C}^{*}$. The long cohomology sequence above splits again into two parts

$$
\begin{aligned}
0 \longrightarrow \mathbf{Z} & \longrightarrow \mathbf{C} \longrightarrow \mathbf{C}^{*} \longrightarrow 1 \\
0 \longrightarrow H^{1}(M ; \mathcal{O}) / H^{1}(M ; \mathbf{Z}) & \longrightarrow H^{1}\left(M ; \mathcal{O}^{*}\right) \longrightarrow H^{2}(M ; \mathbf{Z}) \longrightarrow \cdots
\end{aligned}
$$

By use of the known identification $\operatorname{Pic}(M)=H^{1}\left(M ; \mathcal{O}^{*}\right)$ and by letting

$$
\operatorname{Pic}^{0}(M):=H^{1}(M ; \mathcal{O}) / H^{1}(M ; \mathbf{Z})
$$

one gets

$$
0 \longrightarrow \operatorname{Pic}^{0}(M) \longrightarrow \operatorname{Pic}(M) \longrightarrow H^{2}(M ; \mathbf{Z}) \longrightarrow \cdots .
$$

The topological groups $H^{p}(M ; \mathbf{Z})$ are all discrete because their topology comes from $\mathbf{Z}$. For $p \geq 2, H^{p}(M ; \mathbf{Z})$ are no longer torsion free. Their rank, however, can easily be computed by means of the following lemma. It also turns out that this depends only on the holonomy representation $\rho: \Phi \rightarrow \mathrm{GL}_{\mathbf{Z}}(\Lambda)$ and not on the cohomology class defining (1).

Lemma 2.2. If $G$ is a Bieberbach group as above and $\rho: \Phi \rightarrow \mathrm{GL}_{\mathbf{Z}}(\Lambda)$ the holonomy representation associated with (1), then one has

$$
H^{i}(G ; \mathbf{Q})=\left[\wedge^{i} H^{1}(\Lambda ; \mathbf{Q})\right]^{\Phi},
$$

the $\Phi$-invariant elements of $i$-th exterior power of $H^{1}(\Lambda ; \mathbf{Q})$.

Proof. The $E_{2}$-page of the Lyndon-Hochshild-Serre spectral sequence associated with (1) is given by $E_{2}^{j, i}=H^{j}\left(\Phi ; H^{i}(\Lambda ; \mathbf{Q})\right)$. Because $\mathbf{Q}$ is divisible, everything collapses except the $i$-axis. Therefore

$$
H^{i}(G ; \mathbf{Q}) \simeq H^{0}\left(\Phi ; H^{i}(\Lambda ; \mathbf{Q})\right) \simeq\left[H^{i}(\Lambda ; \mathbf{Q})\right]^{\Phi} .
$$

Since $\Lambda$ is a finitely generated free abelian group and $\mathbf{Q}$ a trivial $\Lambda$-module, by the universal coefficient theorem $H^{i}(\Lambda ; \mathbf{Q}) \simeq \wedge^{i} H^{1}(\Lambda ; \mathbf{Q})$, where $H^{1}(\Lambda ; \mathbf{Q}) \simeq$ $\operatorname{Hom}\left(\Lambda \otimes_{\mathbf{Z}} \mathbf{Q}, \mathbf{Q}\right)$, because $\Phi$ acts trivially on $\mathbf{Q}$.

If $\Omega^{p}$ denotes the sheaf of germs of holomorphic $p$-forms on $M$, the Hodge Decomposition Theorem for any Kähler manifold, see for example [5, page 116], asserts that $H^{i}(M ; \mathbf{C}) \simeq \oplus_{p+q=i} H^{p}\left(M ; \Omega^{q}\right)$, where $H^{p}\left(M ; \Omega^{q}\right)=\overline{H^{q}\left(M ; \Omega^{p}\right)}$. As an immediate consequence, see for example [5, page 314], one has

Lemma 2.3. If $M$ is a n-dimensional, complex, compact, connected, flat manifold, then we have

$$
\operatorname{dim}_{\mathbf{C}} H^{1}(M ; \mathcal{O})=\frac{1}{2} \operatorname{rank}_{\mathbf{Z}} H^{1}(M ; \mathbf{Z})
$$

and

$$
\operatorname{Pic}^{0}(M)=H^{1}(M ; \mathcal{O}) / H^{1}(M ; \mathbf{Z})
$$

is a complex torus. 
The dimension of $\operatorname{Pic}^{0}(M)$ is given by

\section{Lemma 2.4 .}

$$
\operatorname{dim}_{\mathbf{C}} \operatorname{Pic}^{0}(M)=\frac{1}{2} \operatorname{rank}_{\mathbf{Z}}\left[H^{1}(\Lambda ; \mathbf{Z})\right]^{\Phi},
$$

where $\Lambda, \Phi$ are as in (1).

Proof. By the Poincaré Duality $H^{n}(M ; \mathbf{Z}) \simeq H_{0}(M ; \mathbf{Z})$, and because $M$ is connected, $H_{0}(M ; \mathbf{Z}) \simeq \mathbf{Z}$. It follows that $H_{n-1}(M ; \mathbf{Z})$ is torsion free. For

$$
H^{n}(M ; \mathbf{Z}) \simeq \operatorname{Hom}\left(H_{n}(M ; \mathbf{Z}), \mathbf{Z}\right) \oplus \operatorname{Ext}\left(H_{n-1}(M ; \mathbf{Z}), \mathbf{Z}\right),
$$

and $\operatorname{Ext}\left(H_{n-1}(M ; \mathbf{Z}), \mathbf{Z}\right)$ is isomorphic to the torsion subgroup of $H_{n-1}(M ; \mathbf{Z})$. By Poincaré Duality once more, one concludes that $H^{1}(M ; \mathbf{Z})$ has no torsion. The proof now follows from the universal coefficient theorem and Lemma 2.2.

We now want to study the connecting homomorphism $\delta: H^{n}\left(M ; \mathcal{O}^{*}\right) \rightarrow$ $H^{n+1}(M ; \mathbf{Z})$. The discussion that follows is true for every $n$ but we are interested in the case $n=1$ only. $H^{2}(M ; \mathcal{O})$ is a vector space, and $H^{2}(M ; \mathbf{Z}) / \operatorname{Im} \delta \rightarrow H^{2}(M ; \mathcal{O})$ is injective by exactness. Thus, $H^{2}(M ; \mathbf{Z}) / \operatorname{Im} \delta$ is a finitely generated abelian torsion-free group, and we can write

$$
H^{2}(M ; \mathbf{Z}) \simeq H^{2}(M ; \mathbf{Z}) / \operatorname{Im} \delta \oplus \operatorname{Im} \delta .
$$

Moreover (3) implies that $\operatorname{Tors}\left(\operatorname{Pic}(M) / \operatorname{Pic}^{0}(M)\right)=\operatorname{Tors}\left(H^{2}(M ; \mathbf{Z})\right)$. Furthermore, exploiting the topological structure of the groups in $(3)$, if $\left(H^{1}\left(M ; \mathcal{O}^{*}\right)\right)_{0}$ is the identity component of $H^{1}\left(M ; \mathcal{O}^{*}\right)$, we have $\delta\left(\left(H^{1}\left(M ; \mathcal{O}^{*}\right)\right)_{0}\right)=0$ because $H^{2}(M ; \mathbf{Z})$ is discrete. We can also go in the other direction, since

$$
\operatorname{Ker}\left[\delta: H^{1}\left(M ; \mathcal{O}^{*}\right) \rightarrow H^{2}(M ; \mathbf{Z})\right]=e^{*}\left(H^{1}(M ; \mathcal{O})\right)
$$

is connected (because $H^{1}(M ; \mathcal{O})$ is a complex vector space) containing the group $\left(H^{1}\left(M ; \mathcal{O}^{*}\right)\right)_{0}$. We have thus proved

Proposition 2.5. i) The $\operatorname{Im} \delta: H^{1}\left(M ; \mathcal{O}^{*}\right) \rightarrow H^{2}(M ; \mathbf{Z})$ is a direct summand of $H^{2}(M ; \mathbf{Z})$ and contains all its torsion elements. Moreover, $\operatorname{Pic}(M) / \operatorname{Pic}^{0}(M)$ is discrete and

$$
\text { Tors }\left(\frac{\operatorname{Pic}(M)}{\operatorname{Pic}^{0}(M)}\right)=\operatorname{Tors}\left(H^{2}(M ; \mathbf{Z})\right) \text {. }
$$

ii) $\operatorname{Ker} \delta: H^{1}\left(M ; \mathcal{O}^{*}\right) \rightarrow H^{2}(M ; \mathbf{Z})$ is precisely the identity component $\left(H^{1}\left(M ; \mathcal{O}^{*}\right)\right)_{0}$ of $H^{1}\left(M ; \mathcal{O}^{*}\right)$.

Although $H^{2}(M ; \mathbf{Z})$ may not be torsion-free, one has

Proposition 2.6. $\operatorname{rank}_{\mathbf{Z}} H^{2}(M ; \mathbf{Z})=\operatorname{rank}_{\mathbf{Z}}\left[H^{2}(\hat{M} ; \mathbf{Z})\right]^{\Phi}$.

Proof. From the Lyndon-Hochschild-Serre spectral sequence of (4.1.1), we have

$$
\begin{gathered}
H^{2}(M ; \mathbf{Z})=E_{\infty}^{2,0} \oplus E_{\infty}^{1,1} \oplus E_{\infty}^{0,2}, \\
E_{\infty}^{2,0}=E_{3}^{2,0}=E_{2}^{2,0} / \operatorname{Im}\left(E_{2}^{0,1} \rightarrow E_{2}^{2,0}\right), \\
E_{\infty}^{1,1}=E_{3}^{1,1}=\operatorname{Ker}\left(E_{2}^{1,1} \rightarrow E_{2}^{3,0}\right), \\
E_{\infty}^{0,2}=E_{4}^{0,2}=\operatorname{Ker}\left(E_{3}^{0,2} \rightarrow E_{3}^{3,0}\right) .
\end{gathered}
$$


Now, the groups $E_{2}^{2,0}=H^{2}\left(\Phi ; H^{0}(\hat{M} ; \mathbf{Z})\right)=H^{2}(\Phi ; \mathbf{Z})$ and $E_{2}^{1,1}=H^{1}\left(\Phi ; H^{1}(\hat{M} ; \mathbf{Z})\right)$ are finite, thus

$$
\begin{gathered}
\operatorname{rank}_{\mathbf{Z}} H^{2}(M ; \mathbf{Z})=\operatorname{rank}_{\mathbf{Z}} E_{\infty}^{0,2}=\operatorname{rank}_{\mathbf{Z}} E_{4}^{0,2}, \\
E_{3}^{3,0}=E_{2}^{3,0} / \operatorname{Im}\left(E_{2}^{1,1} \rightarrow E_{2}^{3,0}\right)
\end{gathered}
$$

with $E_{2}^{3,0}=H^{3}\left(\Phi ; H^{0}(\hat{M} ; \mathbf{Z})\right)=H^{3}(\Phi ; \mathbf{Z})$ a finite group.

We thus have

$$
\operatorname{rank}_{\mathbf{Z}} H^{2}(M ; \mathbf{Z})=\operatorname{rank}_{\mathbf{Z}} E_{\infty}^{0,2}=\operatorname{rank}_{\mathbf{Z}} E_{3}^{0,2}
$$

where $E_{3}^{0,2}=\operatorname{Ker}\left(E_{2}^{0,2} \rightarrow E_{2}^{2,1}\right)$ with $E_{2}^{2,1}=H^{2}\left(\Phi ; H^{1}(\hat{M} ; \mathbf{Z})\right)$ a finite group. It follows that

$$
\begin{gathered}
\operatorname{rank}_{\mathbf{Z}} H^{2}(M ; \mathbf{Z})=\operatorname{rank}_{\mathbf{Z}} E_{\infty}^{0,2}=\operatorname{rank}_{\mathbf{Z}} E_{3}^{0,2} \\
=\operatorname{rank}_{\mathbf{Z}} E_{2}^{0,2}=\operatorname{rank}_{\mathbf{Z}}\left[H^{2}(\hat{M} ; \mathbf{Z})\right]^{\Phi}
\end{gathered}
$$

\section{The PicARD GRoup}

Keeping the same notation as above $V$ is the universal covering of $M$ and $\hat{M} \equiv$ $V / \Lambda$ is a complex torus which is a finite holomorphic covering of $M$. If $M$ is a flat algebraic manifold, then so is $\hat{M}$.

Associated with (1) one has the cohomology class $c \in H^{2}\left(\Phi ; \Lambda^{\rho}\right)$ that classifies the extension and which we assume to be non-trivial. If $i: \Lambda \rightarrow \Lambda \otimes \mathbf{z} \mathbf{R}=V$ is the map defined by sending $\lambda \mapsto \lambda \otimes 1, \lambda \in \Lambda$, then the class $i_{*}(c) \in H^{2}(\Phi ; V)$ is trivial, for $\Phi$ is finite and $V$ is divisible. We choose

$$
\hat{c}(g)=\frac{1}{|\Phi|} \sum_{h \in \Phi} c(g, h)
$$

since $\Phi$ is always finite, the above definition makes sence. It is not difficult to show that $\hat{c} \in H^{1}(\Phi, V)$ is a "canonical" choice, amongst several ones, of a 1-cocycle such that

(5)

$$
\begin{aligned}
(\delta \hat{c})\left(g_{1}, g_{2}\right) & =\hat{c}\left(\theta\left(\left(g_{1}, g_{2}\right)\right)\right) \\
& =\hat{c}\left(g_{1}\left(g_{2}\right)-\left(g_{1} g_{2}\right)+\left(g_{1}\right)\right)=g_{1} \hat{c}\left(g_{2}\right)-\hat{c}\left(g_{1} g_{2}\right)+\hat{c}\left(g_{1}\right) \\
& =\frac{1}{|\Phi|} \sum_{h \in \Phi} g_{1} c\left(g_{2}, h\right)-\frac{1}{|\Phi|} \sum_{l \in \Phi} c\left(g_{1} g_{2}, l\right)+\frac{1}{|\Phi|} \sum_{k \in \Phi} c\left(g_{1}, k\right) \\
& =\frac{1}{|\Phi|}\left(\sum_{h \in \Phi} g_{1} c\left(g_{2}, h\right)-\sum_{h \in \Phi} c\left(g_{1} g_{2}, h\right)+\sum_{h \in \Phi} c\left(g_{1}, g_{2} h\right)\right) \\
& =\frac{1}{|\Phi|} \sum_{h \in \Phi}\left(g_{1} c\left(g_{2}, h\right)-c\left(g_{1} g_{2}, h\right)+c\left(g_{1}, g_{2} h\right)\right) \\
& =\frac{1}{|\Phi|} \sum_{h \in \Phi} c\left(g_{1}, g_{2}\right)=c\left(g_{1}, g_{2}\right),
\end{aligned}
$$

for $c \in H^{2}(\Phi, \Lambda)$, and thus

$$
g_{1} c\left(g_{2}, h\right)+c\left(g_{1}, g_{2} h\right)=c\left(g_{1}, g_{2}\right)+c\left(g_{1} g_{2}, h\right)
$$

for all $g_{1}, g_{2}, h \in \Phi ; \theta$ is the second differential in the bar resolution and $\delta$ the corresponding differential from the 1-cochains to the 2-cochains.

The elements of $G$ are being represented as the elements of the cartesian product $\Lambda \times \Phi$, where the addition is being defined by

$$
(\lambda, g)+\left(\lambda^{\prime}, g^{\prime}\right)=\left(\lambda+g \cdot \lambda^{\prime}+c\left(g, g^{\prime}\right), g g^{\prime}\right),
$$

for all $\lambda, \lambda^{\prime} \in \Lambda, g, g^{\prime} \in \Phi$.

The action of $G$ on $V, G \times V \rightarrow V$, is given by

$$
(\lambda, g)(z)=g(z)+|\Phi|(\lambda+\hat{c}(g)),
$$


which induces the action of $\Phi$ on $\hat{M}, \Phi \simeq G / \Lambda \times V / \Lambda \rightarrow V / \Lambda$, given by

$$
g[z]=[g(z)+|\Phi|(\lambda+\hat{c}(g))]=[g(z)],
$$

since $|\Phi| \hat{c}(g) \in \Lambda$ (recall that $V=\Lambda \otimes_{\mathbf{z}} \mathbf{R}$ inherits its $G$-module structure from $\Lambda$ ).

For any $G$-module $A$ the action of $\Phi$ on $H^{n}(\Lambda ; A)$ is defined by means of the action of $G$ on $H^{n}(\Lambda ; A)$,

$$
G \times H^{n}(\Lambda ; A) \rightarrow H^{n}(\Lambda ; A),
$$

given by

$$
(\gamma * c)\left(\lambda_{1}, \ldots, \lambda_{n}\right)=\gamma \cdot c\left(-\gamma+\lambda_{1}+\gamma, \ldots,-\gamma+\lambda_{n}+\gamma\right)
$$

for $\gamma \in G$ and $\lambda_{i} \in \Lambda$. For $n=1$ and $A \equiv \mathcal{O}^{*}$, the $G$-group of non-vanishing complex-valued holomorphic functions on $V$, this gives us

$$
\Phi \times H^{1}\left(\Lambda ; \mathcal{O}^{*}\right) \rightarrow H^{1}\left(\Lambda ; \mathcal{O}^{*}\right)
$$

as follows

$$
\left(g * e_{u}\right)(z)=e_{g^{-1} u}\left(g^{-1} z\right),
$$

where $e \in H^{1}\left(\Lambda ; \mathcal{O}^{*}\right), g \in \Phi, u \in \Lambda$, and $z \in V$. One can easily see that the skew, R-bilinear form $F$ which is integral on $\Lambda$ and invariant under the complex structure is $\Phi$-invariant if and only if its associated hermitian form $H, H(x, y)=$ $F(t x, y)+i F(x, y)$, is $\Phi$-invariant. Moreover, starting with a semicharacter $a$ for a $\Phi$-invariant $H$ we can define $\hat{a}(u)=\sqrt[|\Phi|]{\prod_{g \in \Phi} a\left(g^{-1} u\right)}$ which is easily checked to be $\Phi$-invariant. The following is now immediate:

Lemma 3.1. If the $\mathbf{R}$-bilinear form $F$ which is integral on $\Lambda$ and invariant under the complex structure is $\Phi$-invariant, then so is the 1-cocycle

$$
e_{u}(z)=\hat{a}(u) \exp \left\{\pi H(z, u)+\frac{\pi}{2} H(u, u)\right\},
$$

where $u \in \Lambda, z \in \Lambda \otimes \mathbf{z} \mathbf{R}, H(x, y)=F(t x, y)+i F(x, y)$ and $a$ is a semicharacter for $H$.

We can now show

Proposition 3.2. Let $H \in[N S(\hat{M})]^{\Phi}, F=\operatorname{Im} H$, and a be a semicharacter for H. Define

$$
h_{(\lambda, g)}(z): \equiv f_{|\Phi|(\lambda+\hat{c}(g)}(z)
$$

where $f_{u}(z)=e_{-u}(z)=e_{u}^{-1}(z-u)$ and $e_{u}(z)=\hat{a}(u) \exp \left\{\pi H(z, u)+\frac{\pi}{2} H(u, u)\right\}$, $u, \lambda \in \Lambda, z \in V, g \in \Phi$. Then $h \in H^{1}\left(G ; \mathcal{O}^{*}\right)$. Furthermore, the Chern class of the line bundle defined by $h$ is $F$.

Proof. First, we have seen that $\hat{a} \in\left[\operatorname{Pic}^{0}(\hat{M})\right]^{\Phi}$ and by Lemma 3.1, $e \in[\operatorname{Pic}(\hat{M})]^{\Phi}$. One easily sees that

$$
f_{u+v}(z)=f_{v}(z-u) f_{u}(z) .
$$

The cocycle condition for $h$ is as follows:

$$
h_{(\lambda, g)+\left(\lambda^{\prime}, g^{\prime}\right)}(z)=(\lambda, g) \cdot h_{\left(\lambda^{\prime}, g^{\prime}\right)}(z) h_{(\lambda, g)}(z) .
$$


We then have

$$
\begin{aligned}
& h_{(\lambda, g)+\left(\lambda^{\prime}, g^{\prime}\right)}(z) \quad=\quad h_{\left(\lambda+g \lambda^{\prime}+c\left(g, g^{\prime}\right), g g^{\prime}\right)}(z) \\
& =\quad f_{|\Phi|\left(\lambda+g \lambda^{\prime}+c\left(g, g^{\prime}\right)+\hat{c}\left(g g^{\prime}\right)\right)}(z) \\
& \stackrel{(5)}{=} \quad f_{|\Phi|\left(\lambda+\hat{c}(g)+g \lambda^{\prime}+g \hat{c}\left(g^{\prime}\right)\right)}(z) \\
& \stackrel{(7)}{=} \quad f_{|\Phi|\left(g \lambda^{\prime}+g \hat{c}\left(g^{\prime}\right)\right)}(z-|\Phi|(\lambda+\hat{c}(g))) f_{|\Phi|(\lambda+\hat{c}(g))}(z) \\
& \stackrel{((5),(6))}{=} \quad f_{|\Phi|\left(g \lambda^{\prime}+g \hat{c}\left(g^{\prime}\right)\right)}\left(z-|\Phi|\left(\lambda+g c\left(g^{-1}, g\right)-g \hat{c}\left(g^{-1}\right)\right)\right) \\
& \cdot f_{|\Phi|(\lambda+\hat{c}(g))}(z) \\
& f \stackrel{\operatorname{Pic}(\hat{M})]^{\Phi}}{=} f_{|\Phi|\left(\lambda^{\prime}+\hat{c}\left(g^{\prime}\right)\right)}\left(g^{-1} z-|\Phi|\left(g^{-1} \lambda+c\left(g^{-1}, g\right)-\hat{c}\left(g^{-1}\right)\right)\right) \\
& =\quad h_{\left(\lambda^{\prime}, g^{\prime}\right)}\left((\lambda, g)^{-1} \cdot z\right) h_{(\lambda, g)}(z) \\
& \cdot f_{|\Phi|(\lambda+\hat{c}(g))}(z) \\
& =(\lambda, g) \cdot h_{\left(\lambda^{\prime}, g^{\prime}\right)}(z) h_{(\lambda, g)}(z)
\end{aligned}
$$

(where we recall that $(\lambda, g)^{-1}=\left(-g^{-1} \lambda-c\left(g^{-1}, g\right), g^{-1}\right)$ ). For the second part of the proof, first notice that the line bundle $L(H, a)$ corresponding to $H$ and $a$ as above is obtained as the quotient of $\mathbf{C} \times V$ for the action of $\pi_{1}(M)=G$ given by

$$
\phi_{(\lambda, g)}(u, z)=\left(f_{|\Phi|(\lambda+\hat{c}(g))}(z) \cdot u, g z+|\Phi|(\lambda+\hat{c}(g))\right),
$$

where the notation is being kept as above. To find the Chern class of $L(H, a)$ we look at the commutative diagram

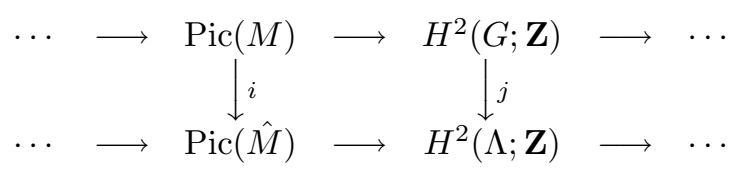

The vertical maps are inclusions. In particular,

$$
j\left(c_{1}(L(H, a))\right)=c_{1}(i(L(H, a)))=c_{1}(i(h))=c_{1}(f)=F .
$$

$c_{1}(L(H, a))$ is a torsion free element of $H^{2}(G ; \mathbf{Z})$, thus by the proof of Proposition 2.6

$$
c_{1}(L(H, a)) \in E_{\infty}^{0,2}<E_{2}^{0,2}=\left[H^{2}(\Lambda ; \mathbf{Z})\right]^{\Phi} .
$$

Since $j$ is the inclusion, it follows that $c_{1}(L(H, a)) \in[\mathrm{NS}(\hat{M})]^{\Phi}$, and this completes the proof.

For any $(\lambda, g) \in G$ the translation $\tau_{(\lambda, g)}: M \rightarrow M$ is homotopic to the identity. Thus, by immitating the standard proof for abelian varieties, one can have further:

Lemma 3.3. Let $L_{1}$ and $L_{2} \in \operatorname{Pic}(M)$ such that $c_{1}\left(L_{1}\right)$ and $c_{1}\left(L_{2}\right)$ are torsion free. Then $c_{1}\left(L_{1}\right)=c_{1}\left(L_{2}\right)$ if and only if there exists a $(\lambda, g) \in G$ such that the one line bundle is the translate of the other, i.e. $L_{1}=\tau_{(\lambda, g)}^{*} L_{2}$.

Proposition 2.5 says that $\operatorname{Pic}^{0}(M)$ is precisely the identity component of $\operatorname{Pic}(M)$. Furthermore, $\operatorname{Pic}(M) / \operatorname{Pic}^{0}(M)$ is finitely generated abelian and therefore discrete as a Lie group. We may, thus, write

$$
\frac{\operatorname{Pic}(M)}{\operatorname{Pic}^{0}(M)} \simeq \operatorname{Tors}\left(H^{2}(M ; \mathbf{Z})\right) \oplus \mathbf{Z}^{N} .
$$

Now, let $\phi: \operatorname{Pic}(M) \rightarrow \frac{\operatorname{Pic}(M)}{\operatorname{Pic}^{0}(M)}$ be the identification map.

Define $\Gamma \equiv \phi^{-1}\left(\operatorname{Tors}\left(H^{2}(M ; \mathbf{Z})\right)\right.$; then $\Gamma$ occurs in the following two extensions:

$$
0 \longrightarrow \operatorname{Pic}^{0}(M) \longrightarrow \Gamma \longrightarrow \operatorname{Tors}\left(H^{2}(M ; \mathbf{Z})\right) \longrightarrow 0
$$


and

$$
0 \longrightarrow \Gamma \longrightarrow \operatorname{Pic}(M) \longrightarrow \mathbf{Z}^{N} \longrightarrow 0 .
$$

The 2-cocycle in $H^{2}\left(\operatorname{Tors}\left(H^{2}(M ; \mathbf{Z})\right)\right.$; $\left.\operatorname{Pic}^{0}(M)\right)$ that classifies extension $(9)$ is trivial. In the opposite case $\operatorname{Pic}^{0}(M)$ would be a subgroup of finite index in a connected Lie group, and so it contradicts Proposition 2.5. Since $\operatorname{Pic}(M)$ is an abelian topological group, we can write

$$
\operatorname{Pic}(M) \simeq \operatorname{Pic}^{0}(M) \oplus \operatorname{Tors}\left(H^{2}(M ; \mathbf{Z})\right) \oplus \mathbf{Z}^{N} .
$$

This, for a compact abelian topological group, follows directly from a theorem of Pontrjagin [12, Theorem 55, page 213]. The case of a general abelian topological group $A$ with identity component a Lie group $A_{0}$ such that $A / A_{0}$ be finitely generated, can be reduced to that of a compact abelian topological group through the following reasoning: Let $\phi: A \rightarrow A / A^{0} \simeq \Phi \oplus \mathbf{Z}^{n}$ be the identification map. Define $G_{1} \equiv \phi^{-1}(\Phi)$. Then $G_{1} \triangleleft A, A / G_{1} \simeq \mathbf{Z}^{n}$ and $A \simeq G_{1} \times \mathbf{Z}^{n}$. Now, $G_{1}$ is a Lie group with finitely many components, so it has a maximal compact subgroup, say $K$; see for example [9]. $K \triangleleft G_{1}$ with $G_{1}$ abelian, so $G_{1} / K \simeq \mathbf{R}^{l}$ and finally $G_{1} \simeq K \times \mathbf{R}^{l}$. The decomposition now follows from Pontryagin's theorem on $K$. Proposition 3.2 and extension (10) combine to give the following version of the Appell-Humbert Theorem for flat manifolds:

Theorem 3.4. If $M$ is a compact, projective, flat manifold, and $\Gamma$ the extension of $\operatorname{Tors}\left(H^{2}(M ; \mathbf{Z})\right)$ by $\operatorname{Pic}^{0}(M)$ as above, then there is a short exact sequence as follows

$$
0 \longrightarrow \Gamma \longrightarrow \operatorname{Pic}(M) \longrightarrow[\mathrm{NS}(\hat{M})]^{\Phi} \longrightarrow 0 .
$$

Furthermore, if

$$
\operatorname{Pic}(M) \simeq \operatorname{Pic}^{0}(M) \oplus \operatorname{Tors}\left(H^{2}(M ; \mathbf{Z})\right) \oplus \mathbf{Z}^{N},
$$

then $N$ is the rank of $[\mathrm{NS}(\hat{M})]^{\Phi}$.

With an eye towards projective embeddings for flat varieties, we notice that the Grothendieck's spectral sequence [6, page 202] yields

$$
H^{0}(M ; \mathcal{O}(L))=\left[H^{0}(\hat{M} ; \mathcal{O}(\hat{L}))\right]^{\Phi} .
$$

The following equivariant version of Lefschetz's theorem is an immediate implication of this remark, Proposition 3.2 and the standard Lefschetz's theorem, see [13, Theorem 5, page 130].

Corollary 3.5. Let $H$ be a $\Phi$-invariant hermitian form on $V$, integral on $\Lambda$, and $L(H, \psi)$ the line bundle on $\hat{M}$ associated to $(H, \psi), \psi$ a semicharacter for $H$. Then $H$ is positive definite if and only if the map induced by a linear system of $\Phi$-invariant holomorphic sections of $L^{\otimes n}$ gives an imbedding of $M$ as a closed submanifold in a projective space for each $n \leq 3$.

\section{ACKNowledgement}

We wish to express our gratitude to Dr. F.E.A. Johnson for suggesting the problem to us and for many interesting conversations, much help and good advice. We extend, further, our sincere thanks to the Institut Fourier, Grenoble, France, in whose warm and hospitable atmosphere most of this paper was written. 


\section{REFERENCES}

[1] Bieberbach, L: Über die Bewegungsgruppen der Euklidischen Räume I; Math. Ann. 70 (1911), 297.

[2] Bieberbach, L: Über die Bewegungsgruppen der Euklidischen Räume II; Math. Ann. 72 (1912), 400.

[3] Charlap, L.S: Compact flat Riemannian manifolds I; Ann. of Math. 81 (1965), 15. MR 30:543

[4] Godement, R: Topologie algébrique et théorie des faisceaux; Hermann, Paris, 1958. MR 21:1583

[5] Griffiths, P and Harris, J: Principles of algebraic geometry; Wiley-Intersience, 1978. MR 80b: 14001

[6] Grothendieck, A: Sur quelques points d' algèbre homologique; Tôhoku Math. J. 9, no.3 (1957). MR 21:1328

[7] Johnson, F.E.A: Flat algebraic manifolds; LMS Lecture Notes, vol. 150, 73-91. MR 93k:32064

[8] Lang, S: Introduction to algebraic and abelian functions; Graduate Texts in Mathematics, vol. 89, Springer-Verlag, 1982. MR 84m:14032

[9] Mostow, G.D: Self-adjoint groups; Annals of Math. 62 (1955), 44-55. MR 16:1088a

[10] Mumford, D: Abelian Varieties; Oxford University Press, 1985. MR 44:219

[11] Nomizu, K: Lie groups and differential geometry; The Mathematical Society of Japan, Tokyo, 1956. MR 18:821d

[12] Pontrjagin, L: Topological groups; Princeton University Press, 1939. MR 1:44e

[13] Weil, A: Introduction à l' étude des variétés Kählériennes; Hermann, Paris, 1958. MR 22:1921

[14] Wolf, J.A: Spaces of constant curvature; McGraw-Hill, 1967. MR 36:829

Rijksuniversiteit Groningen, Afdeling Wiskunde en Informatica, Postbus 800, 9700 AV, Groningen, Netherlands

Current address: 59 Parnithos Street, Vrilissia, 15235 Athens, Greece 